\title{
Observational Constraints on Dust Disk Lifetimes: Implications for Planet Formation
}

\author{
By Lynne A. Hillenbrand \\ ${ }^{1}$ California Institute of Technology, MS 105-24, Pasadena, CA 91105, USA
}

\begin{abstract}
Thus far our impressions regarding the evolutionary time scales for young circumstellar disks have been based on small number statistics. Over the past decade, however, in addition to precision study of individual star/disk systems, substantial observational effort has been invested in obtaining less detailed data on large numbers of objects in young star clusters. This has resulted in a plethora of information now enabling statistical studies of disk evolutionary diagnostics. Along an ordinate one can measure disk presence or strength through indicators such as ultraviolet/blue excess or spectroscopic emission lines tracing accretion, infrared excess tracing dust, or millimeter flux measuring mass. Along an abscissa one can track stellar age. While bulk trends in disk indicators versus age are evident, observational errors affecting both axes, combined with systematic errors in our understanding of stellar ages, both cloud and bias any such trends. Thus detailed understanding of the physical processes involved in disk dissipation and of the relevant time scales remains elusive. Nevertheless, a clear effect in current data that is unlikely to be altered by data analysis improvements is the dispersion in disk lifetimes. Inner accretion disks are traced by near-infrared emission. Moderating a generally declining trend in near-infared continuum excess and excess frequency with age over $<1$ to $8 \pm 4 \mathrm{Myr}$, is the fact that a substantial fraction of rather young $(<1 \mathrm{Myr}$ old) stars apparently have already lost their inner accretion disks while a significant number of rather old (8-16 Myr) stars apparently still retain inner accretion disks. The age at which evidence for inner accretion disks ceases to be apparent for the vast majority $(\sim 90 \%)$ of stars is in the range 3-8 Myr. More distant, terrestrial zone dust is traced by mid-infrared emission where sufficient sensitivity and uniform data collection are only now being realized with data return from the Spitzer Space Telescope. Constraints on mid-disk dissipation and disk clearing trends with radius are forthcoming.
\end{abstract}

\section{Introduction}

A long standing paradigm for the formation of stars, and subsequently planets, involves the rotating collapse of a molecular cloud core to form on a time scale of $\sim 10^{5} \mathrm{yr}$ a central proto-star surrounded by an infalling envelope and accreting disk. Typical ages of revealed young T Tauri and Herbig Ae/Be stars are $\sim 10^{6}$ yr. Gradual dispersal of the initially optically thick circumstellar material occurs in the early pre-main sequence phase as the system evolves through the final stages of disk accretion, which can last $\sim 10^{7} \mathrm{yr}$ or more in at least some well known cases (TW Hya, Hen 3-600, TWA 14 - Muzerolle et al. 2000, 2001 and Alencar \& Batalha 2002; PDS 66 - Mamajek et al. 2002; ECha J0843.3-7905 Lawson et al. 2002; St 34 - White \& Hillenbrand 2005).

Physical processes occurring in younger disks include viscous accretion onto the central star, mass loss due to outflow, irradiation by the central star, ablation due to the stellar wind, turbulent mixing of material, stratification, and gradual settling of the dust towards the disk mid-plane - this last process a critical and limiting step in the path towards planet formation in the standard core accretion model (e.g. Weidenschilling et al. 1997, 2000; Pollack et al. 1996). The total disk mass decreases and the dust:gas mass ratio, assumed at least initially to be in the interstellar ratio, changes with time due to a combination of the above effects. Similarly, the dust particules are assumed interstellarlike in their composition and structure. Of particular interest here is the expected loss of dust opacity due to assembly of small particles into larger bodies that might later 



FIGURE 1. Images of disks at various evolutionary stages scaled to a time line showing our general understanding of the basic phenomena. Data are courtesy of J. Stauffer and B. Patten (left panel, Ori 114-426 optically thick "silhouette disk" as imaged with HST/WFPC), Kalas \& Jewitt 1995 (middle panel, $\beta$ Pic as imaged by a ground-based coronagraph), and P. Kalas (right panel, our own zodiacal dust disk along with a comet, as photographed from Calar Alto).

be known as planetesimals. For solar-type stars, the ultimate result in at least $10 \%$ and perhaps as many as $50 \%$ of cases is a mature solar system (see Marcy, this volume).

In parallel with the discovery and study of exo-solar planets and planetary systems over the last decade (the topic of this conference), we have had dramatic observational confirmation in this same time period of the basic paradigm for star formation as briefly outlined above. Direct images and interferometric observations which spatially resolve young circumstellar disks at optical, near-infrared, and millimeter wavelengths have become common, though are far from ubiquitous. When combined with measured spectral energy distributions, such spatially resolved data are valuable for breaking model degeneracies and thus improving our understanding of source geometry and dust characteristics.

Rough correlation of the spatially resolved and SED appearances of a source, which indicate circumstellar status, with stellar evolutionary state, or age, has long been advocated (e.g. Lada 1987). However, it remains unclear whether the established sequence of circumstellar evolutionary states corresponds directly with source age. White \& Hillenbrand (2004) argue for the Class I/II stages that this is not necessarily the case given the similarities in the stellar photospheric and accretion properties of Class I and II stars as inferred from high dispersion spectroscopy of a large sample in Taurus-Auriga. Likewise, Kenyon \& Hartmann 1995 discuss the Class II/III distribution in the HR diagram, which is indistinguishably intermingled and therefore suggestive of similar ages. Because of uncertainties in age assignments, particularly for the most enshrouded sources which typically do not have ages estimated independent of their circumstellar characteristics, the time scales associated with the dispersal of circumstellar material and the formation of planets are only vaguely constrained at best.

How, then, do we catalog young circumstellar disks and characterize their evolution? 
Disk diagnostics come in two forms: those that trace the dust and those that trace the gas. Dust implies small particles with typical tracers sensitive to sizes less than $1 \mathrm{~mm}$. These include continuum spectral energy distributions over several decades in wavelength, solid state spectroscopic features in the mid-infrared, and direct images measuring either thermal emission at long wavelengths (mid-infrared through millimeter) or scattered light at shorter wavelengths (optical and near-infrared). Gas tracers should reveal the bulk of the mass, at early stages more than $99 \%$ of the total mass if interstellar abundances can be assumed. Sensitive gas observations of young circumstellar disks are, however, limited thus far, restricted to trace species, and dominated by upper limits. Yet recent observations of $\mathrm{CO}, \mathrm{H}_{2}$, and $\mathrm{H}_{2} \mathrm{O}$ seem promising for characterization of terrestrial zone gas. Najita (this volume) presents our knowledge of gas disk evolution in detail.

In addition to academic interest in disk dissipation mechanisms, the main motivation for understanding disk evolution time scales is the relation to planet formation. It seems prudent then to begin with a summary of the capacity of young disks to form planets. We will then continue with methods for assessing the probability that young disks do indeed form planets.

\section{The Potential for Planet Formation in Young Circumstellar Disks}

What are the initial conditions in young disks and what is the likelihood that they are in fact proto-planetary? The raw material of planetary embryos, Earth-like rocks, and Jupiter-like gas giants is indeed abundant, if not ubiquitous, in young disks. But whether any individual disk will form planets is of course unknowable. What we can say is that many of the disks we observe are at least capable of forming planetary systems similar to our own, as evidenced from measured disk sizes, masses, and composition/chemistry. However, as detailed below, the mean disk properties are not yet known due to sensitivity limitations and therefore comparisons to our own proto-solar system based on existing data may be biased.

Disks around young stars were spatially resolved for the first time at millimeter wavelengths (e.g. Sargent \& Beckwith 1987) which measure cold dust and gas in the outer disk regions. Unequal axial ratios, combined with implied dust masses large enough that the central stars should not be optically visible if the dust geometry is spherically symmetric, stood as the strongest evidence for close to a decade of disks surrounding young stars. Further, kinematic models of spatially resolved $\mathrm{CO}$ emission demonstrated consistency with Keplerian rotation (e.g. Koerner et al. 1993; Mannings et al. 1997; Simon et al. 2000; Qi et al. 2003).

Continued interferometric work (e.g. Lay et al. 1994, Dutrey et al. 1996, Duvert et al. 2000; Kitamura et al. 2002; Qi et al. 2003, Semenov et al. 2005). suggested that disk diameters - in instances where spatially resolved, as opposed to point-like, images are obtained - range from $\sim 70-700 \mathrm{AU}$ and are even as large as $\sim 2000 \mathrm{AU}$ in some cases. These disk size estimates are consistent with those inferred from optical/near-infrared scattered light or silhouette images (e.g. McCaughrean \& O'Dell 1996; Padgett et al 1999; Bally et al. 2000), and in the typical case are comparable to or larger than the orbit of the outermost gas giant in our solar system, Neptune. Surface density profiles, e.g. simple power-laws with $\Sigma(r) \propto r^{-p}$ or viscous disk "similarity solutions" with $\Sigma(r) \propto$ $r^{-p} e^{-r^{(2-p)}}$, have suggested a wide range in the value of $\mathrm{p}$ (0-1.5 for the power-law case).

Disk masses are derived from optically thin millimeter flux and an adopted opacitywavelength relationship which leads to uncertainties of factors of 5-10 in disk masses. Under common assumptions the calculated dust masses range from $10^{-4.5}$ to $10^{-3} \mathrm{M}_{\odot}$ (e.g. Beckwith et al. 1990). Making the further assumption that the dust:gas ratio by 
mass is unaltered from the canonical interstellar value of 1:100, total disk masses average around $0.02 \mathrm{M}_{\odot}$, or about the Minimum Mass Solar Nebula (Kusaka et al. 1970; Weidenschilling 1977), the reconstitution of present-day solar system mass and composition to solar consistency. It should be stressed that detection at all of millimeter flux is made amidst an increasing number of upper limits measured for stars with other indicators of disks at shorter wavelengths, and so the true "mean mass" is even lower than that quoted above.

The composition of both young primordial and older debris disks has been shown to resemble that of solar system comets. Ground-based 10 and $20 \mu \mathrm{m}$ work on samples of brighter sources (e.g. Hanner et al. 1995, 1998; Sitko et al. 1999; van Boekel et al 2003; Kessler-Silacci et al 2005) and especially ISO 2-30 $\mu \mathrm{m}$ spectroscopy (e.g. Meeus et al. 2001; Bouwman et al. 2001) have revealed an impressive suite of solid state (and $\mathrm{PAH})$ dust features. Mineralogical details of the dust are modeled on a case-by-case basis due to cosmic variance, but the mean composition appears to be $\sim 70-80 \%$ amorphous magnesium-rich olivines, $\sim 1-10 \%$ crystalline forsterite, $\sim 10-15 \%$ carbons, $\sim 3-5 \%$ irons, and other trace components such as silicas. In particular, crystallinity is advocated in $\sim 10 \%$ of sources.

In summary, the observed sizes, masses, and chemical composition of young disks are all consistent with solar nebula estimates. This is a weak statement, however, since the mean disk properties are biased at present by detection limits and selection effects.

\section{Questions Concerning "Primordial" Dust Disk Evolution}

The term "primordial" is used in reference to disks that are remnants of the star formation process. As outlined above, such disks are composed of dust and gas which participated in the gravitational collapse that formed the star and now comprise the raw materials for the formation of planets. The size, mass, and composition parameters of known young primordial disks are consistent with those estimated for the proto-solar system disk. Terrestrial planets and the rocky cores of giant planets originate from disk dust while the gaseous envelopes of giant planets originate with the disk gas. Primordial disks are in the process of dissipating through either planet formation or one of the other disk dispersal mechanisms mentioned earlier.

It is instructive to point out that primordial disks are physically distinguished from the so-called "debris" disks, which are secondary rather than primordial. These are gas-poor disks, comprised of dust which is regenerated during and subsequent to the growth of planets as the large/massive bodies incite collisions amongst smaller bodies to re-form dust. Debris disks, like primordial disks, are in the process of dissipating, though via a different mechanism. Rather than sticking collisions which result in smaller particles growing to become larger particles (and eventually becoming undetectable via thermal infrared radiation), debris disk particles experience shattering collisions and gradually grind themselves down to the point at which grains are efficiently removed from the system via effects such as Poynting-Robertson drag and stellar winds. However, new dust is continually being generated in the cascade generated by collisions between the larger bodies and the dissipating evolution is punctuated by the infusion of new material in the debris cascade.

The collisional history in the inner solar system, due to the influence of the outer giant planets on such debris, is well-documented in the cratering records on the Moon and Mars. These records indicate to some degree the evolution of the cratering rate and the large body size distribution with time. We have no firm record of the dust evolution in solar system, but even today there is "debris dust" found/assumed in the Asteroid and 
Kuiper belt regions. Because of the strong theoretical connection between debris dust and planetary perturbers, there is much interest in the debris belts seen around stars other than the Sun (see Meyer, this volume) whose evolution we can study by investigating samples of different age.

Here I focus on the properties and evolution of dust in primordial disks. For any given disk, the dust mass is expected to decrease with time throughout the duration of the planet-building process, perhaps over tens of Myr. Then, if planets have successfully formed, the dust mass increases at the on-set of the debris disk phase before slowly declining again with time over many Gyr.

To understand the process of planet formation we must understand how quantities such as initial disk size and radial/vertical structure, initial disk mass and mass surface density, and the initial disk composition and chemistry all evolve with time and, further, the relative importance of various disk dispersal mechanisms (e.g. accretion, ablation, grain growth as mentioned above). Over what time scales are dust (and gas) detectable and how does the mass ratio of dust:gas evolve? What physical parameters determine disk longevity? What is the frequency of different end states, in particular of planetary configurations? Most important for understanding the rarity or commonality of the formation of our own solar system, what is the mean and the dispersion in all of the above distributions?

As we continue to develop the tools for answering these questions we can also consider several pertinent "second parameter" issues. One category of these relate to properties of the central star. Are there correlations in initial disk properties or disk evolution diagnostics with stellar properties such as the radiation field (particularly x-ray and ultraviolet output), stellar mass, or system metallicity, all of which may have important effects on disk structure and chemistry? A second category of second parameters are those related to disk physics effects. How does disk accretion history, in particular poorly understood outburst phenomena such as FU Ori or EXOr type events affect disk evolution? Thirdly, what is the role of environment? Multiplicity in the form of binary, triple, or quadruple systems can influence disk evolution when the companions are within or just exterior to the disk. Clustered versus isolated star forming environments in which effects such as increased ionization or photo-evaporation of disk material by massive stars, dynamical effects due to high stellar density, or the mechanical effects of multiple jets/outflows, could be important for disk evolution. Consequently, understanding of multiplicity statistics in the form of frequency and orbital parameters and clustering statistics in the form of spatial density and luminosity function is important for our appreciate of the range of plausible disk evolutionary paths.

In summary, there are many parameters considered potentially influential in the disk evolution process. The only way to probe effectively disk evolution and its many dependencies is through the assembly of sufficient statistics over the appropriate range of ages and "second parameter" conditions. This is a tall order indeed, but a road down which we have at least started.

\section{Enough Questions - What do we Know and How do we Know it?}

The disk dispersal time or disk lifetime is often asserted in the literature as "about $10 \mathrm{Myr}$ ". This estimate is certainly good to an order of magnitude, but the justification for this number, or any other specific number, is weak at best given the data in hand. Some inner dust/gas disks have disappeared within $1 \mathrm{Myr}$, by the time the star becomes optically visible. Some inner dust/gas disks last at least $10 \mathrm{Myr}$. In at least one case, that of our own solar system, the need has been expressed by some theorists for the 
TABLE 1. Dust disk properties measurable as a function of stellar age

\begin{tabular}{lll}
\hline Property & Observational Diagnostic & Example Study \\
\hline Disk geometry & Interferometry; SED modelling & Eisner et al 2004, 2005 \\
Mean excess in SED; disk fraction & Broad-band photometry & Hillenbrand et al. 2006; Mamajek et al 2004 \\
Accretion rate on to star & Ultraviolet/optical spectrophotometry & Muzerolle et al 2000; White \& Hillenbrand 2004 \\
Dust mass & Millimeter/sub-millimeter photometry & Carpenter et al. 2005; Wyatt et al. 2003 \\
Dust mineralogy; size distribution & Mid-infrared spectroscopy & Kessler-Silacci et al 2005; van Boekel et al 2005 \\
\hline
\end{tabular}

gas disk to survive $100 \mathrm{Myr}$ or longer, in order to form the outermost gas giants. As astronomers, we want to understand the mean and the dispersion in the lifetime time of young primordial disks, both dust and gas. In this section I will review disk diagnostics, appropriate subject samples, and the difficulties involved in assessing stellar ages. In the next section I proceed to summarize what is known about disk evolutionary trends.

\subsection{Disk Diagnostics}

To look for evidence of disk evolution in action we need to consider carefully the diagnostic potential of any particular observable. Many are available. However, the information obtained varies widely between different tracers of disk evolution. This is due in part to variation in observational sensitivity, for example as a function of wavelength, and in part to the varying efficacy of different disk tracers. In addition, the precision and accuracy of stellar ages - that other, often under-scrutinized or even ignored axis in any disk evolution diagram - needs to be critically assessed.

Deferring sensitivity considerations for the time being, what can we hope to measure as a function of stellar age? Resolved disk images as discussed in my introductory comments certainly have led to a wider appreciation of the convincing case for primordial "protoplanetary" disks. In fact, it is not an over-statement to say that the stunning images from ground-based interferometers (millimeter) and from the Hubble Space Telescope (optical and near-infrared) were responsible for transforming the field of star formation from a following of dedicated and knowledgeable disciples to high profile science. However, the reality is that few such spatially resolved images exist at present. Study of most young disk systems relies, for the most part, on so-called indirect measurements such as broadband photometry and high resolution optical or near-infrared spectroscopy.

Table 1 details several properties of quantitative interest for young circumstellar disks and the observational diagnostics used to measure them. These are generalized properties and each can be broken down into a more detailed set of specific physical characteristics. There is an increasingly large literature on these topics and I list only a few example studies. In most categories there is some, but limited, evidence for at least modest evolution from primordial disk conditions. Conclusions in the area of evolution are typically based on samples of young disks ranging from small (a few) to moderate (tens to a few hundred) in size.

As mentioned above, the focus of my discussion will be on dust disk diagnostics. In particular I will focus on disk detection as revealed through infrared excesses, observed emission in excess of that expected from a stellar photosphere. Various levels of sophistication may be employed in the application of this technique, ranging from fully assembled spectral energy distributions covering several decades in wavelength to twocolor diagrams which cover only a limited portion of the excess spectrum to statistical study of the disk fraction (frequency of objects in a given age bin with convincing ev- 
idence of a disk) or mean excess (magnitude or strength of the excess). Full spectral energy distributions covering ultraviolet to millimeter wavelengths have been available for only small samples of well-studied young stellar objects, making statistics difficult to assemble. Two-color diagrams are widely available, enabling statistical studies, but more difficult to interpret without detailed knowledge of 1) more of the spectral energy distribution 2) the intrinsic spectral energy distribution in the absence of reddening which can be prevalent towards young star forming regions and 3) the properties of the underlying star. Disk fraction and mean excess techniques account for both reddening and intrinsic stellar colors, but are based on partial spectral energy distributions. The discussion below will focus on these last techniques.

Data for infrared excess investigations are available most abundantly at near-infrared wavelengths, due to several decades of ground-based work combined with the large and uniform 2MASS photometric database at 1.2, 1.6, $2.2 \mu \mathrm{m}$ now available. Ground-based work at mid-infrared wavelengths has been more limited in both scope and sensitivity; previous space-based platforms were revolutionary at the time, but somewhat similarly limited in sensitivity (IRAS) and scope (ISO). Thus our understanding of disk statistics in the $3-100 \mu \mathrm{m}$ wavelength regime is not as well developed as in the $1-2 \mu \mathrm{m}$ regime. The Spitzer Telescope is currently accumulating sensitive data between 3 and $70 \mu \mathrm{m}$ enabling the construction of mid-infrared spectral energy distributions. These observations focus on many of the historically favored objects, though blind imaging surveys of star forming regions and young open clusters are also being conducted and will provide needed statistics over the next few years.

\subsection{Stellar Samples}

Once a technique is adopted, a sample must be chosen. In order to establish trends, robust, complete, and unbiased samples must be established over an appropriate range of ages. For the problem at hand this includes the youngest revealed protostars through the ages characteristic of star-forming regions still associated with molecular gas $(<1-2$ Myr), and continuing to the entire period of terrestrial and gas giant planet formation (thought to be $\sim 100$ Myr for our own solar system), as depicted schematically in Figure 1.

Young star clusters would appear ideal for these sorts of studies because they provide the needed statistics. Furthermore, clusters have attractive attributes such as the relatively uniform distance, age, and chemical composition of their members, all of which minimize analysis complication. Young star clusters can therefore, in principle, provide the samples required to compare disk properties such as the mean and dispersion in disk lifetimes as a function of stellar mass (within a cluster) and as a function of stellar age or chemical composition (between clusters). However, careful investigation reveals that the young star samples identified to date are lacking with respect to some important issues.

First, known targets for investigations of disk evolution can be segregated into the following four coarse age groups: $<1 \mathrm{Myr}$ (embedded or partially embedded star forming regions), 1-3 Myr (optically revealed stellar populations still associated with molecular gas), 5-15 Myr (association members in gas-poor "fossil" star-forming regions), and finally the punctuated ages (55 Myr, $90 \mathrm{Myr}, 120 \mathrm{Myr}$, and $600 \mathrm{Myr}$ ) of the nearest populous open clusters.

Of concern is that the age distribution of known samples of young stars over the 1$100 \mathrm{Myr}$ age range of interest for disk evolution is not uniform. Ample numbers (many thousands) of young stars associated with regions of recent star formation have been identified through surveys of molecular cloud complexes. Because of the intense focus on stellar census data for these young regions, such stars dominate the total numbers and 
thus bias the available statistics in the overall young star age distribution towards the 1-3 Myr youngest age category.

The statistics decline dramatically at ages older than about 5 Myr and out to about $50 \mathrm{Myr}$, due to a lack of large identified samples with known ages in this "young intermediate" age regime. There are no open clusters or large associations within $150 \mathrm{pc}$ or so of the Sun in the 5-50 Myr age range save the Sco OB-2 association at the upper distance and lower age limit. Field stars 5-50 Myr old are extremely hard to identify since they stand out from much older field star populations only with detailed observations (not, e.g., in wide-field photometric surveys). They may be revealed through signatures of youth such as common proper motion with kinematically young groups, enhanced Li I absorption, Ca II H\&K core emission, and X-ray activity. In fact, finding stars in this age range should be relatively easy due to our circumstance in the Galaxy near a ring of moderately recent star formation ("Gould's Belt"). Yet within $150 \mathrm{pc}$ or so, current samples of 5-50 Myr old stars number only in the few tens, consisting of members of the TW Hya, Beta Pic, Eta Cha, and Tuc/Hor moving groups. Continued correlation of large-scale kinematic and activity databases with sufficient spectroscopic following is beginning to address this deficiency. However, the present lack of ample numbers of young stars in the 5-50 Myr age range serves to increase the error bars in disk evolution diagnostic statistics right where all the most interesting "action" of disk evolution may be taking place.

At ages older than 50 Myr there are again ample samples due to the proximity of several well-studied near-by open clusters. Specifically, the IC2602 / IC2391 pair, Alpha Per, Pleiades, and Hyades clusters, all within 200 pc and well-studied, are benchmark points in any evolutionary diagram involving either stellar or circumstellar properties.

\subsection{Stellar Ages}

A comprehensive discussion of stellar ages is beyond the scope of this review. Suffice it to say that there are large number of age diagnostics, most of which are poorly calibrated in the young pre-main sequence age range of interest here. The most commonly used measure of stellar age in the $<1-30 \mathrm{Myr}$ age range is location in the Hertzsprung-Russell (HR) diagram compared to theoretical predictions of luminosity and temperature evolution as a function of time. HR diagrams are shown in Figure 2 for a number of current and recently star forming regions as well as young open clusters in the solar neighborhood.

HR diagrams can be used to infer a mean age and an apparent age dispersion as a function of effective temperature, for each cluster. One issue to consider is whether the age spreads one would infer for stars in young star clusters from their observed luminosity spreads indeed correspond to age ranges rather than observational errors, the default assumption. Luminosity spreads do decrease with time (e.g. consider the Orion Nebula Cluster vs the Alpha Per Cluster in Figure 2). However, the errors in converting from observables to luminosity are the largest in the young pre-main sequence phase, just where the apparent luminosity spreads are the largest. Thus the conversion from luminosity to age, and the implied age spreads, are confusing. Age spreads, or lack thereof, are important to understand for the purposes of studying evolutionary diagrams since one needs either to consider all stars in a single cluster to have the mean age of the apparent distribution, or to assess ages individually and adopt an age for each star. Because this issue has not been satisfactorily addressed at the young ages of interest here, the evolutionary time scales for young circumstellar disks thus have large random uncertainties depending on whether potentially real age spreads are accounted for in the analysis or not.

Another caution is that theoretical pre-main sequence evolutionary calculations, on 


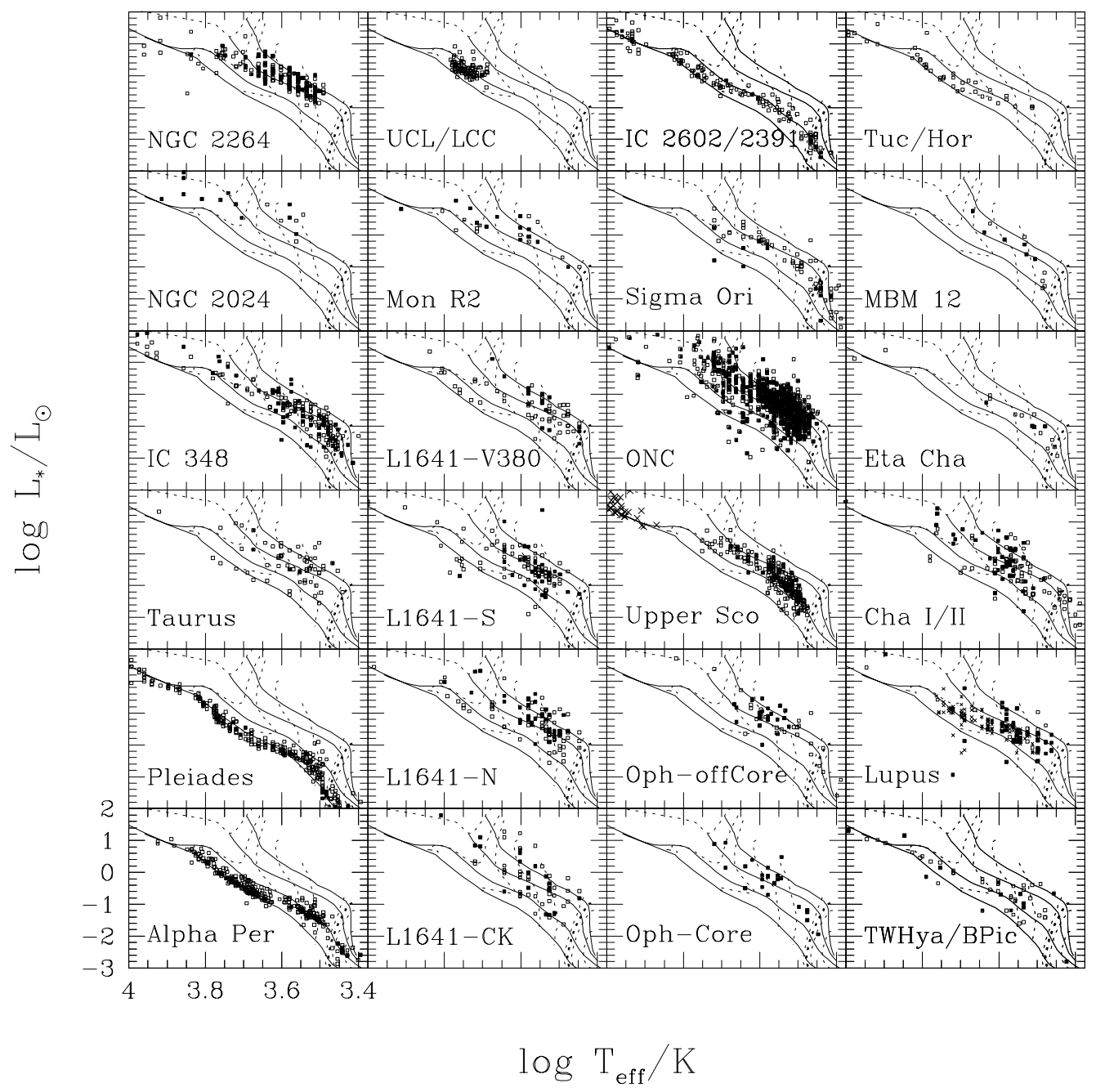

FIGURE 2. HRDs for well-studied star-forming regions and young clusters. Data were placed using the temperature scale, color scale, and bolometric corrections described in Hillenbrand $\&$ White (2004) and a wide variety of literature for the fundamental data. Pre-main sequence evolutionary calculations are those of D'Antona \& Mazzitelli $(1997,1998)$ for isochrones of 0.1, 1.0, 10, and $100 \mathrm{Myr}$ (solid lines) and masses 0.03, 0.06, 0.08, 0.1, 0.2, 0.4, 0.8, 1.5, and $3.0 \mathrm{M}_{\odot}$ (dashed lines).

which age estimates from the HR diagram rely, have significant uncertainties in their predictions. First, there is variation between various theory groups of $20-100 \%$ over certain mass and age ranges (see comprehensive discussion in Baraffe et al. 2002). Second, pre-main sequence calculations thus far do not favor well in comparison to observational constraints. Specifically, they collectively under-predict stellar masses by 30-50\% (Hillenbrand \& White 2004). Further, they under-predict low-mass stellar ages by 30-100\% compared to lithium-depletion boundary estimates and over-predict high-mass stellar ages by 20-100\% compared to post-main sequence evolutionary calculations. Because of this lack of theoretical validation of the age calibration of pre-main sequence isochrones, 
the evolutionary time scales for young circumstellar disks thus have large systematic uncertainties.

\section{Disk Evolution}

I describe now the observational constraints on the evolution of potentially protoplanetary disks through the disk clearing phase. As already emphasized I will focus on dust disk evolution, mentioning gas where it should not be forgotten, but not discussing gas in any detail. Three spatial regimes in the disk are considered: inner disk dissipation traced by near-infrared continuum data, mid-disk dissipation traced by mid-infrared data, and outer disk dissipation traced by millimeter wavelength data.

\subsection{Inner Disk Dissipation}

There is a well-demonstrated empirical connection between accretion and outflow diagnostics measured by high dispersion optical spectroscopy which probes the kinematics of warm gas in the vicinity of young stars (e.g. Hartigan et al. 1995; White \& Hillenbrand 2004). A similar empirical connection (e.g. Hartigan et al. 1990, Kenyon \& Hartmann 1995) exists between the same spectroscopic emission lines and the blue continuum excess measured as spectroscopic veiling, both signatures of accretion directly onto the star, and photometric near-infrared $(1-3 \mu \mathrm{m})$ continuum flux excess arising in the innermost $(<0.05-0.1 \mathrm{AU})$, and thus hottest, disk regions. These correlations affirm the basic connection between accretion from a disk and ejection in an outflow.

Furthermore, both the spectroscopic signatures of accretion and the near-infrared excess are separately demonstrated to correlate inversely with stellar age, over small age ranges. Detailed modeling of the accretion temperature, density, velocity, and geometric structure is required to convert emission line strengths and profiles into mass accretion rates. More common than emission line profile studies is the measurement from high dispersion spectroscopy of continuum veiling which can also be converted to a mass accretion rate after making assumptions about the bolometric correction to derive a total accretion luminosity, and about the infall geometry.

Treatments of the trends in the accretion rate with age have been presented by Muzerolle et al. 2000 and Calvet et al. 2005a. At least several stars appear to show measurable accretion signatures beyond 10 Myr. Existing trends have been inferred by considering the individually derived ages of stars based on the HR diagram. They are thus subject to the criticism that age spreads in individual clusters such as Taurus, Chamaeleon, or the TW Hydra association may be overestimated and that comparisons between the mean accretion rates and mean stellar ages in each cluster cluster may be more appropriate. Similar criticisms are also levied below against treatments of near-infrared excess behavior with age.

For the near-infrared continuum analysis, we utilize measured flux above expected photospheric values to infer disk presence. Increasingly complex inner disk geometries have been advanced over the last decade (e.g. Mahdavi \& Kenyon 1998), which complicates the expectations regarding the magnitude of a near-infrared excess given constant other parameters for the star and the disk. In the analysis discussed here we do not consider such geometric complications and assess simply whether there is, or is not, evidence for disk emission at near-infrared wavelengths for our sample.

In calculating the color excess due to the disk, one must make two corrections from observed colors. First, it is necessary to derive and subtract the contribution from foreground or large scale circumstellar extinction. Second, from the remaining color, a correction for the underlying stellar photosphere is performed in order to arrive at the intrinsic 

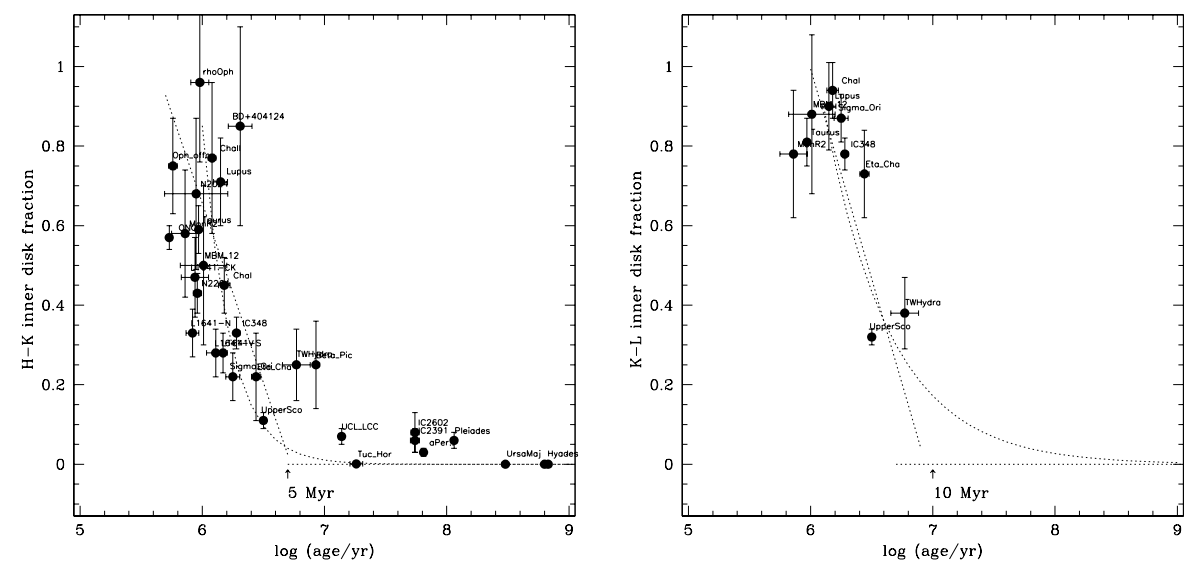

FIGURE 3. Inner accretion disk fraction vs. stellar age inferred from $\mathrm{H}-\mathrm{K}$ excess (left panel) and K-L excess (right panel) measurements, binned by cluster or association. All young stars which we are able to locate in the HR diagram based on information in the literature (about 3500) and having inferred masses $0.3-1.0 \mathrm{M}_{\odot}$ are included in this figure. Individual clusters are treated as units of single age corresponding to the median age inferred from the HR diagram. A cut of $\Delta(\mathrm{H}-\mathrm{K})>0.05 \mathrm{mag}$ is used to define a disk. Standard deviation of the mean (abscissa) and Poisson (ordinate) error bars are shown. The linear and exponential fits were derived for ages $<30$ Myr; the linear fit has negative slope close to unity with rms 0.3 .

color excess due to the disk. In formulaic terms, using $\mathrm{H}-\mathrm{K}$ color as an example, the disk excess is quantified as $\Delta(H-K)=(H-K)_{\text {observed }}-(H-K)_{\text {reddening }}-(H-K)_{\text {photosphere }}$. Similar indices can be derived for J-K or K-L colors which also probe inner disk regions though sense dust at slightly different temperatures. In order to effect the above extinction and photospheric corrections, and hence assess intrinsic color excesses, several different sets of information are required: 1) a spectral type, for intrinsic stellar color and bolometric correction determination, 2) optical photometry, for dereddening and locating stars on the HR diagram, assuming known distance, and 3) infrared photometry, for measurement of disk "strength".

It should be borne in mind that disk strength, quantified as above from measurement of the absolute value of the infrared excess, is still a relative quantity. For any given star/disk system the infrared excess is affected by both stellar properties (mass, radius) and disk properties (accretion rate, inclination, geometry). Meyer et al. 1997 and Hillenbrand et al. 1998 (both in collaboration with Calvet) provide detailed discussions of these dependencies apropos near-infrared excesses. The effects of stellar and disk parameters on overall spectral energy distributions are discussed more comprehensively by D'Alessio et al. (1999).

Now what about that pesky other axis of stellar age? Instead of discussing in detail all of the inherent uncertainties in locating stars in the HR diagram (Figure 2), and in inference of stellar ages and masses from those diagrams, I will simply assume fiducial cluster ages based on the median apparent age of stars in the mass range $0.3-1.0 \mathrm{M}_{\odot}$. With both a disk diagnostic and a method of cluster age estimation we can now explore the evidence for disk evolution.

Our best effort at empirically measuring the time scale for the evolution of inner circumstellar accretion disks is represented in Figure 3, produced from a sample of $\sim 3000$ stars located $\sim 50-500 \mathrm{pc}$ from the Sun. To be included in the sample each star was required to have the spectral type, optical photometry, and infrared photometry necessary 
for calculation of $\Delta(H-K)$ or $\Delta(K-L)$, as described above. It should be noted that there are far fewer stars with available L-band photometry than available (J)HK photometry. There are several important points made by these example plots.

First, even at the earliest evolutionary stages at which stars can be located in the HR diagram, the optically thick inner disk fraction does not approach unity. There are several well-known examples of objects near the stellar birthline without any evidence for disks. This may be influenced by selection effects in that protostars and objects in transition from the protostellar to the optically revealed stage generally lack the spectroscopic data required for inclusion in our sample. However, the result is more apparent in the $\mathrm{H}$ $\mathrm{K}$ excess figure than in the K-L excess figure. If a real effect (as opposed to an effect introduced by bias in the samples selected for L-band photometry), this indicates strongly that some disk evolution does happen very early on for some stars, before they become optically visible.

Second, beyond $1 \mathrm{Myr}$ of age existing samples are less biased by complications of extinction and self-embeddedness, and hence more representative of underlying stellar populations as a whole (if not close to complete for most of the regions plotted). At these ages, there is a steady decline with time in the fraction of stars showing near-infrared excess emission (i.e. optically thick inner disks), as well as large scatter at any given age. We will return to the issue of the scatter later. The conversion of diagrams like Figure 3 into a frequency distributions of accretion disk lifetimes is the next step, and really what we want to know rather than disk frequency with age; this analysis is presented in Hillenbrand, Meyer, \& Carpenter (2006).

Third, the median lifetime of inner optically thick accretion disks based on assessment of modern data may be as short as 2-3 Myr with essentially no evidence for HKL excess present in the median star beyond 5 Myr. Clearly there are exceptions such as the noted cases of $\sim 10$ Myr old accretion disks.

Other discussions of inner disk lifetimes have used different techniques and more limited samples of stars (e.g. Walter et al. 1988, Strom et al. 1989, Skrutskie et al. 1990, Beckwith et al. 1990, Strom 1995; Haisch et al. 2001). As with most scientific inquiries, the results derived depend on the details of both the samples and the analysis. Within the proposed random and systematic uncertainties, all of the above studies are comparable in their results. Previous general conclusions regarding inner disk lifetimes in the 3-10 Myr age range are, broadly speaking, similar to our findings of $<2-3$ Myr for the evolution of the mean disk. Further, although most disks appear to evolve relatively rapidly, a small percentage appear to retain proto-planetary nebular material for factors of 5-10 longer than does the average disk.

\subsection{Mid-Disk Dissipation}

As emphasized above, near-infrared wavelengths measure hot dust in the innermost disk regions, the presence of which is well correlated empirically with independent (spectroscopic) evidence for accretion onto the star. Because only a small amount of dust is required to make the inner disk optically thick, near-infrared continuum excesses tell us little about the bulk of the disk mass or surface area, which radiates at much cooler temperatures and hence longer wavelengths. Further, because the dynamical time is a function of radius in the disk, there is some expectation in the scenario that disk dissipation involves sticking collisions that eventually generate planetesimals, for disks to evolve in the inner regions first and the outer regions later (e.g. Hollenbach et al 2000). Thus studying disk frequency with age (or better yet, disk lifetime) as a function of disk radius is of great interest. 




Figure 4. Terrestrial zone disk fraction vs. stellar age inferred from N-band excess measurements for $\sim 50$ stars, taken from Mamajek et al 2004.

Mid-infrared wavelengths, $\sim 10-90 \mu \mathrm{m}$, probe disk radii $\sim 1-5 \mathrm{AU}$, equivalent to the outer terrestrial and inner gas giant planetary zones of our solar system. To date, observational sensitivity has been the primary hindrance to measurement of disk evolution at these wavelengths. The sensitivity required at mid-infrared wavelengths is in fact orders of magnitude more in flux density units than that needed in the near-infrared due to the Rayleigh-Jeans fall-off of the stellar photosphere. Despite the large number of nondetections or upper limits, previous mid-infrared observations of small samples of young stars have revealed evolutionary trends.

The most recent statistical results using ground-based equipment (e.g. Mamajek et al 2004; Metchev et al 2004), when considered in the same excess fraction format as Figure 3 , show similar morphology with $\sim 10 \mathrm{Myr}$ needed for depletion of $90 \%$ of optically thick terrestrial zone dust. Figure 4 is reproduced from Mamajek et al. 2004. The implication is that the terrestrial zone disk dissipation times are perhaps consistent with, or at most factors of a few longer than, inner disk dissipation times. If true, the combined near- and mid-infrared results suggest that disk evolution is both rapid and relatively independent of radius. However, as was true in the analysis of inner disk lifetimes, a decreasing fractional excess that is never unity is suggestive of a dispersion in disk lifetimes, in this case over an order of magnitude in age.

The Spitzer Telescope offers dramatic improvement to heretofore available mid-infrared continuum excess probes of dust evolution. Spitzer is sensitive to nearby stellar photospheres between 3.5 and at least $24 \mu \mathrm{m}$ with additional sensitive capability out to $70 \mu \mathrm{m}$. Spitzer thus enables meaningful statistical studies of primordial (and debris - see Meyer, this volume) disk evolution within and beyond the terrestrial planet zone. Advances over the previous IRAS/ISO and ground capability are already revolutionizing the field. Restricting the discussion to only $8 \mu \mathrm{m}$ results, Silverstone et al. (2006) study both field stars and cluster / association members $<30$ Myr old, Young et al (2004) present results for a single $30 \mathrm{Myr}$ old cluster NGC 2547, and Stauffer et al (2005) discuss the $120 \mathrm{Myr}$ old Pleiades cluster. All of these papers reaffirm the basic Mamajek findings that terrestrial zone dust is depleted within $10 \mathrm{Myr}$, but add needed statistics. Further Spitzer results are forthcoming.

At longer wavelengths, 25-60 $\mu \mathrm{m}$, data from the IRAS and ISO satellites were even more limited in addressing disk evolution problems, again due to the sensitivity require- 
ments of such investigations. These platforms were not capable of detecting the stellar photospheres of young stars at the necessary $150 \mathrm{pc}$ distance. However, some results at $60 \mu \mathrm{m}$ have been presented in the same form as Figure 3 (e.g. Meyer \& Beckwith 2000; Robberto et al. 1999), again suggesting consistency with the Mamajek et al results at 10 $\mu \mathrm{m}$. However, Spangler et al. (2001) and Habing et al. (2001) argue, based on ISO data, for a much longer mid-infrared disk dissipation time scale, on the order of hundreds of Myr. There may be some confusion in these two studies between primordial and debris disks as a single, continuous evolutionary path is not expected over this long time scale (see, Decin et al. 2003 for a critical assessment).

Again, Spitzer will revolutionize the field due to its increased sensitivity and spatial resolution over previous capabilities. Results at $24 \mu \mathrm{m}$ for the $5 \mathrm{Myr}$ old Upper Sco association (Chen et al. 2005) and for the 10 Myr TW Hya association (Low et al. 2005) have appeared thus far. However, the mix of spectral types in these early studies relative to the roughly solar-type stars discussed above make rigorous comparisons of the disk dissipation statistics with radius premature.

\subsection{Outer Disk Dissipation}

Moving outward in wavelength and hence downward in temperature, millimeter wavelength emission probes the cold outer ( 50-100 AU) disk regions and is optically thin. Most millimeter observations (e.g. Andrews \& Williams 2005) have been directed towards stars younger than $\sim 10^{7}$ year, but because of the distance of these populations, generally place only upper limits on dust masses beyond the youngest phase (e.g. Duvert et al. 2000). Recent application of a clever technique to push below formal detection limits has resulted in more stringent constraints on the typical disk masses in several very young clusters (Carpenter 2002; Eisner \& Carpenter 2003), finding mean dust masses of $5 \times 10^{-5} \mathrm{M}_{\odot}$ (which can be augmented by an assumed gas-to-dust ratio to infer a total mass). Because of the more dispersed nature of older stars, there remain few such stringent constraints on typical dust masses in the 3-10 Myr old age range.

Dust mass surveys of older $\left(10^{7}-10^{9}\right.$ year), closer, candidate debris disk stars (e.g. Zuckerman \& Becklin 1993, Jewitt 1994, Wyatt et al. 2003, Carpenter et al. 2005) also reveal mostly upper limits due to current sensitivity challenges, but also several detections of very proximate stars with dust masses as low as $10^{-8} \mathrm{M}_{\odot}$. In an analysis of the ensemble of upper limits, Carpenter et al. (2005) find marginal evidence for continuous evolution in the dust masses at expected primordial disk ages, from the 1-2 Myr young clusters to the 3-10 and 10-30 Myr field stars, which may in fact have already transitioned from primordial to debris disks.

Assessment of primordial disk evolution at radii of several tens to hundreds of $\mathrm{AU}$, where the bulk of the disk mass resides, thus awaits dramatically improved millimeter and sub-millimeter sensitivity. Such is on the horizon with the comissioning of CARMA and ALMA.

\section{How Long Does the Dissipation Process Take, Once Initiated?}

Once the process of disk dissipation starts, how long does it take for an individual object to transition from optically thick to optically thin dust? The expectation is for a short transition, based on calculations of initial grain growth via pairwise sticking collisions followed by runaway growth that forms large planetesimals (Moon-sized) on times scales of only $\sim 10^{5}$ yr (e.g. Wetherill \& Stewart 1993; Weidenshilling \& Cuzzi 1993). Is there a radial dependence to the disk clearing or do inner, mid, and outer disk regimes dissipate simultaneously? While there are clear decreasing trends with advancing stellar age both 
in the fraction of objects exhibiting infrared excess and in the mean magnitude of the infrared excess (not addressed in the discussion above), this does not inform us about the disk dissipation time for an individual object. The observed trends and their dispersion can be used, however, to construct statistical arguments that address the duration of the disk dissipation process, as a function of radius.

Historically, a relatively short, less than a few hundred thousand year time scale, has been inferred for the transition from an optically thick circumstellar disk to an optically thin circumstellar disk. The logic is based on two arguments, first the disk statistics in binary pairs, and second the small number (and therefore fraction) of objects found in transition between the optically thick and optically thick stages. Binary pairs, particularly in Taurus, have been well characterized in terms of the well-known CTTS (disked) and WTTS (disk-less) categories. Numerous studies (e.g. Hartigan, Strom, \& Strom 1994, Prato \& Simon 1997, Duchene et al. 1999, Hartigan \& Kenyon 2003) have found that the vast majority, $>80 \%$, of binary pairs are either both CTTS or both WTTS with mixed pairs relatively rare. This argues that the disk dissipation time is shorter than the absolute age difference between the members of stellar binaries.

Concerning transition objects, in the well-studied Taurus star-forming region, for example, V819 Tau and V773 Tau were argued by Skrutskie et al (1990) to be the only two members out of approximately 150 known found with little or no near-infrared excess but small mid-infrared excess $\dagger$, a result confirmed by Simon \& Prato (1995) and Wolk \& Walter (1996). This argument relies on the assumption of cluster coevality. As discussed above, this may not be a valid assumption at the few (2-3) Myr level. Spitzer data presented by Hartmann et al (2005) appear to add several other objects to the "transition" category, such as CIDA 8, CIDA 11, CIDA 12, CIDA 14, DH Tau, DK TauB, and FP Tau.

Yet other Taurus objects have no evidence for excess out to $10 \mu \mathrm{m}$ but substantial excess at longer wavelengths. These are different from the sources detected with excess at or short-wards of $10 \mu \mathrm{m}$, but in transition from having optically thick to optically thin inner disks. They may be even slightly more evolved (in a circumstellar sense). One interpretation is that on the time scale that inner disk clearing has completed, these disks may be transitioning from optically thick to optically thin in the mid or outer disk regions. GM Aur has long been appreciated in this category (e.g. Koerner et al. 1993; Rice et al 2003). Others with excesses only at long wavelengths were not detectable with the sensitivity of IRAS but are being revealed by Spitzer, for example CoKu Tau4 (D'Alessio et al 2005) and DM Tau (Calvet et al 2005b).

Collectively, both the optically thin and the inner cleared disks can be referred to as "transitional." In regions other than Taurus, the case for transitional disks has also been made. For example, Gauvin \& Strom (1992) highlighted CS Cha in Chamaeleon as having a large inner cleared region (tens of AU) but a substantial far-infrared excess indicative of a robust outer disk. Nordh et al. (1996) show 7-15 $\mu \mathrm{m}$ flux ratios in Chamaeleon that are scattered around either the colors expected from flat/flared disks, or around photospheric colors, with essentially no objects located in between these groupings. These observations support the rapid transition time scales argued for Taurus members. Low et al (2005) observed the same effect at longer wavelengths, $24 \mu \mathrm{m}$, in the much older TW Hya association.

In summary, in young $(<3 \mathrm{Myr})$ star forming regions transition disks rare, with most

$\dagger$ see Duchene et al 2003 for evidence concerning the multiplicity of V773 Tau and argument that the apparent excess can be attributed to one of the companions rather than betraying a "fossil" disk. 
stars having circumstellar material that is either consistent with an optically thick disk or not apparent at all. Further, there are specific examples of stars with dust in the terrestrial planet zone (0.1-3 AU) but not in the very inner disk $(<0.05 \mathrm{AU})$. This suggests that material closest to the star may disappear first, as accretion subsides, and that the disk is cleared from the inside out. In slightly older (10 Myr) regions the only disks left appear to be those in transition, already evolved or fully cleared in the inner disk regions but retaining mid-infrared excesses indicative of mid-range disks.

How does disk clearing occur? While photometric studies at infrared and millimeter wavelengths such as those discussed above can provide statistics for assessing the dust disk dissipation time scale, and hence the dust disk lifetime, they tell us very little about the physics of the process. Studies of evolutionary changes in the disk structure or dust grain processes, by contrast, do provide physical insight but are restricted to much smaller samples which can be studied in detail. Spectral energy distributions and mineralogy are two tools that can provide insight.

Typically, grain growth and disk evolution arguments have been made from measurement of the frequency dependence of continuum opacity in the expression $\tau_{\nu}(r)=$ $\kappa_{\nu} \times \Sigma(r)$ where $\kappa_{\nu} \propto \nu^{\beta}$. The $\beta=2$ appropriate for interstellar dust often yields in measurements of optically thin sub-/millimeter spectral energy distributions to $\beta=0-1$ (see Miyake \& Nakagawa, 1993). The effects on the overall spectral energy distribution of grain growth are presented in a parameter study of disk geometry and grain properties by D'Alessio et al. (2001).

Detailed spectral energy distributions are most useful when combined with spatially resolved imaging at one or more wavelengths, enabling degeneracies in model parameters to be removed. Modelling studies of objects in different circumstellar evolutionary stages e.g., Class 0, Class I, Class II, (perhaps even Class III someday) spectral energy distributions can provide constraints on disk geometry. Some examples of such work are the analyses by Wood et al. (1998), Wolf et al. (2003), Eisner et al. (2006), Kitamura et al. (2002), and Calvet et al (2002). It should be borne in mind, however, that the connections between circumstellar and stellar evolutionary states are not yet clear.

As the dust transitions from optically thick to optically thin, perhaps as a function of radius, spectroscopy becomes an especially important tool for assessing grain size distribution and composition. Mineralogical studies reveal information about dust processing, for example changes in chemical composition or mean grain size. There is evidence already for the growth of grains in young disks to sizes larger than are expected based on the assumption that disk grain properties are consistent with those of interstellar dust. Direct probes of grain growth are spectroscopic studies that are sensitive to the opacity from particular species having particular size ranges. Work in the 8-13 $\mu \mathrm{m}$ atmospheric window (e.g. Kessler-Silacci et al. 2005, van Boekel et al. 2005) is being complemented, improved, and extended by Spitzer studies from 5-40 $\mu \mathrm{m}$. Especially compelling observations would be those that can obtain spatially resolved mineralogical information. Intriguing results in this area have emerged recently from the VLTI (e.g. van Boekel et al 2004)

\section{Present Assessment of Dust Clearing Trends with Radius}

A single sentence summary of the above set of results on inner disks, mid-range disks, and outer disks is that the often quoted "10 Myr disk lifetime" is a gross generalization. While there are some clear declining trends at several wavelengths in measured disk strength and disk frequency with time, the simple fact that we can consider the quantity "disk frequency" implies that at any given age some stars have disks while others do not, 
and thus a range in disk evolutionary times. The dispersion in disk lifetimes is at least factors of a few if not an order of magnitude.

There is a some evidence that disk clearing times may be shorter in the near-infrared than in the mid-infrared though this conclusion is not strong at present. The most conservative statement is that dust disk dissipation appears to occur within 3-8 Myr for the vast majority of stars, with minor evidence for more rapid time scales at smaller radii. The dissipation time for the mean disk may be $<2-3$ Myr. Sensitive observations with Spitzer of statistically significant samples of young stars spanning an appropriate age range are needed before such conclusions are robust, however. Such are beginning to emerge.

It should also be noted that the methods employed to date for statistical study of disks and disk lifetimes largely detect the presence or absence of a disk and do not tell us much about the detailed disk properties (radial/vertical structure, total mass, composition, etc.). This is another area in which the improved sensitivity and the spectroscopic capabilities of Spitzer along with the spatially resolved imaging capabilities of ground-based facilities will improve our understanding, though only for selected individual objects.

Finally, we reiterate that a complication in developing our empirical understanding of the time scales and physical processes associated with primordial disk dissipation is that soon after dusty disk material begins agglomerating to form planetesimals, the proto-planets likely collide and re-form the dust. When does a particular system go from being primordial (dominated by growth of smaller bodies into larger ones) to debris (dominated by destruction of larger bodies into smaller ones which are then removed from the system via Poynting-Robertson drag and stellar wind effects)? For disks surrounding stars with ages in the 5-15 Myr age range there is some ambiguity as to whether they are primordial or debris disks. Several prominent examples are TW Hya, which is still accreting (Muzerolle et al 2000), Beta Pic and AU Mic, both of which are nearby and spatially resolved, and new spatially unresolved detections in the 5-15 Myr age range emerging from Spitzer (e.g. Chen et al. 2005; Low et al. 2005; Silverstone et al. 2006).

As in the above discussion of primordial optically thick disks, spatial resolution is the key element for advances in debris disk studies with, for example, the color of scattered light providing critical information about the radial distribution of grain sizes (e.g. Metchev et al 2005). Our main diagnostic for observationally distinguishing primordial from debris disks is the presence of gas, discussed in this proceedings in more detail by Najita.

\section{Implications for Planet Formation}

The discovery of exo-solar planets more than a decade ago made understanding of the connections between disks (both primordial and secondary/debris) and planets more critical than ever. The near ubiquity of circumstellar dust and gas disks around very young stars has been advocated for decades, but only within the past decade uniformly accepted by the astronomical community. The turning point was availability of spatially resolved images of young gaseous and dusty disks at millimeter, sub-millimeter, infrared, and optical wavelengths. Beyond evidence for disks, the detailed information provided by 1) such images, 2) spectral energy distributions sampled over more than four decades in wavelength, and 3) dust and gas spectroscopy, is increasing our understanding of the initial conditions for planet formation. This review has concentrated on dust disk diagnostics.

However, detailed understanding of the processes of star and planet formation requires statistical assessment of global properties and evolutionary trends, in addition to study of 
individual objects. Despite the large amount of data presently available we are only now beginning to achieve the observational sensitivity needed to probe the full range of disk conditions. For the assembly of statistics we still need to rely on traditional photometric and spectroscopic techniques rather than well sampled spectral energy distributions plus spatially resolved imaging at multiple well separated wavelengths, which are available in relatively few cases.

With the statistics available at present, there are constraints on disk dissipation time scales though they are limited in terms of the detail needed to constrain theories. Evidence for decreasing trends with age in the disk fraction, the mean disk accretion rate, and the mean disk mass are apparent. There are also signs in individual young disks of evolution from interstellar grain parameters. What may be most interesting however, is the large dispersion about the mean at any given age, in all of these trends. This in particular speaks to the frequency distribution of paths for solar system formation and evolution.

By establishing the decay with time of primordial dust via near- and mid-infrared excess around stars of different mass, we will take, over the next decade, the first step in understanding the possibilities for planetary formation. Studies to determine the time scales for dust disk dissipation should be followed by those aiming to similarly quantify time scales for gas disk dissipation. Fully constraining the time period over which the raw materials needed for planetary formation are available means, ultimately, following the evolution of disk surface density as a function of radius from the central star. One outstanding problem in planning for this kind of statistically robust future is that we do not have adequate samples of stars in the $5-50 \mathrm{Myr}$ age range, a critical time in planet formation and early solar system evolution.

Various theories of dust settling, planet formation, and planetary migration within disks are discussed elsewhere in these proceedings. The limited constraints from theory are consistent with the equally vague precision with which disk lifetimes can be inferred from observations of potential planetary systems now in the making. Thus the interpretation of observations is not - yet - the limiting step in solidifying our understanding of planet formation.

When, where, and how frequently do planets form in circumstellar disks? How do forming planetary systems evolve dynamically? What is the range in diversity of stable planetary system architectures? How frequent are habitable planets? How unique is our solar system? These are fairly sophisticated questions to be asking, especially so given our only rough knowledge of the planet formation process in our own solar system. Meteoritic evidence concerning survival time of the solar nebula suggests "several Myr" as the relevant evolutionary time scale. Studies, especially those concerning extinct radionuclides, support this time span for initial accretion, differentiation, and core formation (see e.g. review by Wadhwa \& Russell 2000). It should be emphasized that although dispersal of the solar nebula may occur quickly, the total duration over which inner planet formation was completed in fact approached 30-100 Myr.

An overarching goal of these pursuits is to connect what is observed elsewhere with the history of our own solar solar system, and hence enhance our appreciation of the uniqueness - or lack thereof - of it, our Earth, and in some respects the human circumstance.

\section{REFERENCES}

Aikawa, Y., van Zadelhoff, G. J., van Dishoeck, E. F., Herbst, E. 2002, AA, 386, 622

Akeson, R.L., Ciardi, D.R., van Belle, G.T., Creech-Eakman, M.J., \& Lada, E.A. 2000, ApJ, 543,313 
Alencar, S.H.P. \& Batalha, C. 2002, ApJ, 571, 378

Alves, J.F., Lada, C.J., \& Lada, E.A. 2001, Nature, 409, 159

Andrews, S.M. \& Williams, J.P. 2005, ApJ, 631, 1134

Bally, J., O’Dell, C.R.; McCaughrean, M.J. 2000, AJ, 119, 2919

Baraffe, I., Chabrier, G., Allard, F., \& Hauschildt, P.H. 2002, AA, 382, 563

Beckwith, S.V.W., Henning, T., \& Nakagawa, Y. 2000, in Protostars and Planets IV, (eds V. Mannings, A.P. Boss, and S.S. Russell, Univ. Arizona Press), p. 533

Beckwith, S.V.W., Sargent, A.I., Chini, R., \& Gusten, R. 1990, AJ, 99, 924

Bouwmann, J., Meeus, G., de Koter, A., Hony, S., Dominik, C., \& Waters, L.B.F.M. 2001, AA, 375,950

Calvet, N., Briceo, C., Hernndez, J., Hoyer, S., Hartmann, L., Sicilia-Aguilar, A., Megeath, S.T.. \& D'Alessio, P. 2005a, AJ, 129, 935

Calvet, N., D'Alessio, P., Watson, D.M., Franco-Hernndez, R. et al. 2005b, ApJ, 630, 185

Calvet, N., D'Alessio, P., Hartmann, L., Wilner, D., Walsh, A. \& Sitko, M. 2002, ApJ, 568, 1008

Carpenter, J.M. 2002, AJ, 124, 1593

Carpenter, J.M. Wolf, S., Schreyer, K., Launhardt, R., Henning, T. 2005, AJ, 129, 1049

Chen, C.H., Jura, M., Gordon, K.D., \& Blaylock, M. 2005, ApJ, 623, 493

D’Alessio, P., Calvet, N., \& Hartmann, L., Lizano, S., Cant, J. 1999, ApJ, 527, 893

D'Alessio, P., Calvet, N., \& Hartmann, L. 2001, ApJ, 553, 321

D'Alessio, P., Hartmann, L.; Calvet, N., Franco-Hernndez, R., et al. 2005, ApJ, 621, 461

D'Antona, F. \& Mazzitelli, I. 1997, Mem. Soc. Astro. Ital., 68, 807 and on-line at www.mporzio.astro.it/ dantona.

Decin, G., Dominik, C., Waters, L. B. F. M., \& Waelkens, C. 2003, ApJ, 598, 636

Duchene, G., Ghez, A.M., McCabe, C., \& Weinberger, A.J. 2003, ApJ, 592, 288

Duchene, G., Monin, J.-L., Bouvier, J., \& Menard, F. 1999, A\&A, 351, 954

Dutrey, A., Guilloteau, S., Duvert, G., Prato, L., et al. 1996, AA, 309, 493

Duvert, G., Guilloteau, S., Menard, F., Simon, M., \& Dutrey, A. 2000, AA, 355, 165

Eisner, J. A., Hillenbrand, L. A., Carpenter, J.M., \& Wolf, S. 2006, ApJ, in press.

Eisner, J. A., Hillenbrand, L. A., White, R. J., Akeson, R. L., Sargent, A. I. 2005, ApJ, 623, 952

Eisner, J. A., Lane, B. F., Hillenbrand, L. A., Akeson, R. L., Sargent, A. I. 2004, ApJ, 613, 1049

Eisner, J. A.; Carpenter, J.M. 2003, ApJ, 588, 360

Gauvin, L.S. \& Strom, K.M. 1992, ApJ, 385, 217

Habing, H.J., Dominik, C., Jourdain de Muizon, M., Laureijs, R.J., et al. 2001, AA, 365, 545

Haisch, K.E., Lada, E.A., \& Lada, C.J. 2001, AJ, 121, 2065

Hanner, M.S., Brooke, T.Y., \& Tokunaga, A.T. 1995, ApJ, 438, 250

Hanner, M.S., Brooke, T.Y., \& Tokunaga, A.T. 1998, ApJ, 502, 871

Hartigan, P. \& Kenyon, S.J. 2003, ApJ, 583, 334

Hartigan, P., Strom, K.M., \& Strom, S.E. 1994, ApJ, 427, 961

Hartigan, P., Hartmann, L., Kenyon, S.J., Strom, S.E., Skrutskie, M.F. 1990, ApJ, 354, 25

Hartigan, P., Edwards, S., \& Ghandour, L. 1995, ApJ, 452, 736

Hartmann, L., Megeath, S.T., Allen, L., Luhman, K., Calvet, N., D'Alessio, P., FrancoHernandez, R., Fazio, G. 2005, ApJ, 629881

Hillenbrand, L.A., White, R.J. 2004, ApJ, 604, 741

Hillenbrand, L.A., Meyer, M.R., \& Carpenter, J.M. 2006, in preparation

Hollenbach, D.J., Yorke, H.W., \& Johnstone, D. 2000, in Protostars and Planets IV, (eds V. Mannings, A.P. Boss, and S.S. Russell, Univ. Arizona Press), p. 401

Jewitt, D.C. 1994, AJ, 108, 661

Kalas, P. \& Jewitt, D. 1995, AJ, 110, 794

Kenyon, S.J. \& Hartmann, L. 1995, ApJS, 101, 117

Kessler-Silacci, J.E., Hillenbrand, L.A., Blake, G.A., Meyer, M.R. 2005, ApJ, 622, 404

Kitamura, Y., Momose, M., Yokogawa, S., Kawabe, R., Tamura, M., \& Ida, S. 2002, ApJ, 581, 357

Koerner, D.W., Sargent, A.I., \& Beckwith, S.V.W. 1993, Icarus, 106, 2

Kusaka, T., Nakano, T., Hayashi, C. 1970, Prog. Theor. Phys., 44, 1580 
Lada, C. J. 1987, in IAU Symp. 115, Star Forming Regions, ed. M. Peimbert \& J. Jugaku (Dordrecht: Kluwer)

Lay, O.P., Carlstrom, J.E., Hills, R.E., \& Phillips, T.G. 1994, ApJL, 434, 75

Lawson, W.A., Crause, L.A.; Mamajek, E.E., \& Feigelson, E.D. 2002, MNRAS, 329, 29

Low, F.J., Smith, P.S., Werner, M., Chen, C., Krause, V., Jura, M., Hines, D.C. 2005, ApJ, 631, 1170

Mahdavi, A. \& Kenyon, S.J. 1998, ApJ, 497, 342

Mamajek, E. E., Meyer, M. R., \& Liebert, J. 2002, AJ, 124, 1670

Mamajek, E. E., Meyer, M. R., Hinz, P. H., Hoffman, W. F., Cohen, M., \& Hora, J. L. 2004, ApJ, 612, 496

Mannings, V., Koerner, D.W., \& Sargent, A.I. 1997, Nature, 388, 555

McCaughrean, M.J. \& O'Dell, C.R. 1996, AJ, 111, 1977

Meeus, G., Waters, L.B.F.M., Bouwman, J., van den Ancker, M.E., Waelkens, C., \& Malfait, K. 2001, AA, 365, 476

Metchev, S.A. Hillenbrand, L.A., \& Meyer 2004, ApJ, 600, 435

Metchev, S.A., Eisner, J.A., Hillenbrand, L.A., \& Wolf, S. 2005, ApJ,

Meyer, M.R. \& Beckwith, S.V.W. 2000 in "ISO Surveys of a Dusty Universe" (eds. D. Lemke, M. Stickel, and K. Wilke, Springer-Verlag Lecture Notes in Physics), p. 548

Meyer, M.R., Calvet, N., \& Hillenbrand, L.A. 1997, AJ, 114, 288

Miyake, K, \& Nakagawa, Y. 1993, Icarus, 106, 20

Muzerolle, J., Calvet, N., Briceno, C., Hartmann, L., Hillenbrand, L., 2000, ApJL, 535, 47

Muzerolle, J., Hillenbrand, L., Calvet, N., Hartmann, L., Briceo, C. 2001, ASP Conf Ser, 244, 245

Nordh, L., Olofsson, G., Abergel, A., Andre, P., et al. 1996, AA, 315, L185

Padgett, D., Brandner, W., Stapelfeldt, K.R., Strom, S.E., Tereby, S., \& Koerner, D. 1999, AJ, 117,1490

Pollack, J.B., Hubickyj, O., Bodenheimer, P., Lissauer, J.J., et al. 1996, Icarus, 124, 62

Prato, L. \& Simon, M. 1997, ApJ, 474, 455

Qi, C., Kessler, J.E., Koerner, D.W., Sargent, A.I.; Blake, G.A. 2003, ApJ, 597, 986

Rebull, L.M., Makidon, R.B., Strom, S.E., Hillenbrand, L.A., et al. 2002, AJ 123, 1528

Rebull, L.M., Hillenbrand, L.A., Strom, S.E., Duncan, D.K., et al. 2000, AJ, 119, 3026

Rice, W.K.M., Wood, K., Armitage, P.J., Whitney, B.A., \& Bjorkman, J.E. 2003, MNRAS, 342, 79

Robberto, M., Meyer, M.R., Natta, A., \& Beckwith, S.V.W., 1999 in "The Universe as Seen by ISO" (eds. P. Cox and M.F. Kessler, ESA-SP 427), p. 195

Sargent, A.I. \& Beckwith, S.V.W. 1987, ApJ, 323, 294

Sargent, A.I. \& Beckwith, S.V.W. 1991, ApJL, 382, 31

Semenov, D., Pavlyuchenkov, Ya., Schreyer, K., Henning, Th., Dullemond, C., Bacmann, A. 2005 ApJ, 621, 853

Silverstone, M.D., Meyer, M.R., Mamajek, E.E., Hines. D.C. et al. 2006, ApJ, in press

Simon, M., Prato, L. 1995, 450, 824

Simon, M., Dutrey, A., \& Guilloteau, S. 2000, ApJ, 545, 1034

Sitko, M.L., Grady, C.A., Lynch, D.K., Russell, R.W. \& Hanner, M.S. 1999, ApJ, 510, 408

Skrutskie, M.F., Dutkevitch, D., Strom, S.E., Edwards, S., Strom, K.M., Shure, M.A. 1990, AJ 99, 1187

Spangler, C., Silverstone, M., Sargent, A.I., Becklin, E.E., \& Zuckerman, B. 2001, ApJ, 555, 932

Stauffer, J., Rebull, L., Carpenter, J., Hillenbrand, L., et al. 2005, AJ, 130, 1834.

Strom, S.E. 1995, RMAA 1, 317

Strom, K.M., Strom, S., Edwards, S., Cabrit, S., \& Skrutskie, M. 1989, AJ, 97, 1451

van Boekel, R., Waters, L. B. F. M., Dominik, C., Bouwman, J., de Koter, A., Dullemond, C. P., \& Paresce, F. 2003, A\&A, 400, 21

van Boekel, R., Waters, L. B. F. M., Dominik, C., Dullemond, C. P., Tielens, A. G. G. M., \& de Koter, A. 2004A\&A...418..177

van Boekel, R., Min, M., Waters, L. B. F. M., de Koter, A., Dominik, C., van den Ancker, M. E., \& Bouwman, J. 2005 A\&A, 437, 189 
Wadhwa \& Russell 2000, in Protostars and Planets IV, (eds V. Mannings, A.P. Boss, and S.S. Russell, Univ. Arizona Press), p. 995

Walter, F.M., Brown, A., Mathieu, R.D., Myers, P.C., \& Vrba, F.J. 1988, AJ, 96, 297

Weidenschilling, S.J. 1977, ApSS, 51, 153

Weidenschilling, S.J. 2000, SSRv, 92, 295

Weidenschilling, S.J., Spaute, D., Davis, D.R., Marzari, F.; Ohtsuki, K. 1997, Icarus, 128, 429

Weidenschilling, S.J., \& Cuzzi, J.N. 1993, in Protostars and Planets (eds. E.H. Levy and J.I. Lunine, Univ. Arizona Press), p. 1031

Wetherill, G.W. \& Stewart, G.R. 1993, Icarus, 106, 190

White, R.J., Hillenbrand, L.A. 2004, ApJ, 616, 998

White, R.J., Hillenbrand, L.A. 2005, ApJL, 621, 65

Wolf, S., Padgett, D.L., \& Stapelfeldt, K.R . 2003, ApJ, 588, 37

Wolk, S.J. \& Walter, F.M. 1996, AJ, 111, 2066

Wood, K., Kenyon, S.J., Whitney, B., \& Turnbull, M. 1998, ApJ, 497, 404

Wyatt, M.C., Dent, W. R. F., Greaves, J. S., 2003, MNRAS, 342, 876

Young, E. T., Lada, C. J., Teixeira, P., Muzerolle, J., et al. 2004, ApJS, 154, 428

Zuckerman, B. \& Becklin, E. E. 1993, ApJ, 414, 793 